TITLE:

\title{
Stimulation of CK2-dependent Grp94 phosphorylation by the nuclear localization signal peptide.
}

\section{AUTHOR(S):}

Miyata, Yoshihiko; Yoneda, Yoshihiro; Yahara, Ichiro

\section{CITATION:}

Miyata, Yoshihiko ...[et al]. Stimulation of CK2-dependent Grp94 phosphorylation by the nuclear localization signal peptide.. Molecular and cellular biochemistry 2011, 356 (02-1): $191-200$

\section{ISSUE DATE:}

2011-10

URL:

http://hdl.handle.net/2433/152541

\section{RIGHT:}

The final publication is available at www.springerlink.com; この論文は 出版社版でありません。引用の際には出版社版をご確認ご利用くださ $\omega_{\circ}$; This is not the published version. Please cite only the published version. 


\section{Stimulation of CK2-dependent Grp94 phosphorylation}

\section{by the nuclear localization signal peptide}

Yoshihiko Miyata, Yoshihiro Yoneda, Ichiro Yahara

Address for the correspondence:

Y. Miyata

Department of Cell and Developmental Biology, Graduate School of Biostudies, Kyoto University, Kitashirakawa Oiwake-cho, Sakyo-ku, Kyoto 606-8502, Japan

Tel: +81-75-753-4231, FAX: +81-75-753-4235, e-mail: ymiyata@Lif.kyoto-u.ac.jp

Department of Cell Biology, The Tokyo Metropolitan Institute of Medical Science

Y. Yoneda

Department of Frontier Biosciences, Graduate School of Frontier Biosciences, Osaka University

I. Yahara

Department of Cell Biology, The Tokyo Metropolitan Institute of Medical Science

Present address:

I. Yahara

Ina Institute, Medical \& Biological Laboratories Co., Ltd. 
Keywords CK2, Grp94, NLS, nuclear translocation, phosphorylation, kinase

\section{Abbreviations}

Grp94 glucose regulated protein 94

CK2 protein kinase CK2

ER endoplasmic reticulum

NLS nuclear localization signal

Hsp90 heat shock protein 90

Con A concanavalin A

DTT dithiothreitol

EDTA ethylenediaminetetraacetic acid

SDS-PAGE sodium dodecyl sulfate-polyacrylamide gel electrophoresis 
Abstract The nuclear localization signal sequence (NLS) of SV40 Large T antigen is essential and sufficient for the nuclear translocation of the protein. Phosphorylation often modulates the intracellular distribution of signaling proteins. In this study, we investigated effects of the NLS-peptide of Large $\mathrm{T}$ antigen on protein phosphorylation. When crude cell lysates were incubated with $\left[\gamma^{32} \mathrm{P}\right] \mathrm{ATP}$, phosphorylation of several endogenous substrates with molecular masses of $100,80,50$, and $45 \mathrm{kDa}$ by an endogenous kinase was stimulated by the addition of the wild type NLS-peptide (CPKKKRKVEDP). The mutated NLS-peptide (CPKTKRKVEDP) and the reversed NLS-peptide (PDEVKRKKKPC) are weak in the nuclear localization activity, and they only weakly stimulated phosphorylation of these substrates. The mobility of the $100 \mathrm{kDa}$ phosphoprotein was indistinguishable with that of an endoplasmic reticulum (ER)-resident molecular chaperone glucose-regulated protein 94 (Grp94) belonging to the Hsp90 family, and purified Grp94 was phosphorylated by a kinase in cell lysates in an NLS-dependent fashion. The $100 \mathrm{kDa}$ protein was identified as Grp94 by immunoprecipitation and reconstitution experiments. Purification of the NLS-dependent Grp94 kinase by sequential biochemical column chromatography steps resulted in isolation of two polypeptides with molecular masses of $42 \mathrm{kDa}$ and $27 \mathrm{kDa}$, which were identified as $\alpha$ and $\beta$ subunit of protein kinase CK2, respectively, by western blotting analysis and biochemical characterization. Moreover, effect of an excess amount of GTP and V8 peptide mapping showed that the NLS-dependent Grp94 kinase in the cell lysate is identical with CK2. Surprisingly purified CK2 did phosphorylate Grp94 even without the NLS-peptide, suggesting that an additional suppressive factor is required for NLS-dependent phosphorylation of Grp94 by CK2. We suggest a possible general role for CK2-catalyzed phosphorylation in the regulation of NLS-dependent protein nuclear translocation. 


\section{Introduction}

Nuclear localization of a protein in cells is determined by a balance between nuclear import and export of the protein through the nucleopore complexes on nuclear membranes. The transport of proteins between the nucleus and the cytoplasm is a signal-mediated process [1]. The nuclear import and export of proteins are both governed by specific amino acid sequence tags on cargo proteins. The peptide segments found to direct nuclear localization, the nuclear localization signal (NLS) sequences, were first identified in SV40 Large T antigen [2] and nucleoplasmin [3]. The NLS sequences in nuclear proteins are typically short stretches of a single series of basic residues (resembling the NLS of SV40 Large T antigen) or of two clusters of basic residues interrupted by a 10-12-amino acid spacer (bipartite NLS). They appear to be functional in various natural and artificial protein contexts, suggesting that NLS function is largely independent of the secondary/tertiary structure. A single replacement of a lysine (MT-NLS) in the sequence of SV40 Large T antigen NLS abolished the nuclear localization activity of the peptide $[2,4,5]$. In addition, a peptide with the reverse amino acid sequence (RT-NLS) of SV40 Large T antigen NLS shows only a weak NLS activity [6], suggesting that the sequence, but not the composition, of the NLS-peptide is important for the nuclear localization activity.

Large proteins require assistance of nuclear transport factors to traverse the nuclear pore complexes. The NLS is recognized by the specific receptor system that consists of the tag-recognizing transport protein factors. Nuclear import of NLS-containing cargo proteins starts with the formation of a stable complex in the cytoplasm. This NLS-dependent complex consists of two transport factors, importin (karyopherin) $\alpha$ and $\beta[7,8]$. Generally, importin $\alpha$ recognizes the NLS and functions as an adaptor molecule to recruit the cargo on importin $\beta$, resulting a formation of a cargo-importin $\alpha$-importin $\beta$ ternary complex $[7,8]$. Recognition is also mediated by importin $\beta$ or one of its homologues alone. Each member of importin family 
proteins recognizes a unique set of proteins or RNAs, creating multiple transport pathways. In addition, importin $\beta$ can interact with components of the nucleoporins and functions as a carrier molecule [9].

Protein phosphorylation constitutes the pivotal mechanism for the rapid and efficient regulation of the cellular process by inducing structural or functional changes of proteins involved in cellular signal transduction systems. Intimate links between phosphorylation and intracellular localization have been suggested for many signaling proteins [10]. Protein kinases and phosphatases both play key roles in modulating cellular distribution of proteins. It should be important to analyze the relationships between the signal-driven protein phosphorylation and protein nuclear translocation at a molecular level.

Protein kinase CK2 is a ubiquitous and essential protein kinase with pleiotropic substrates and function [11,12], but it remains unclear how CK2 activity is regulated in cells [13]. CK2 recognizes serine and threonine residues in an acidic environment and phosphorylates a broad spectrum of endogenous and artificial substrates involved in many cellular functions $[14,15]$. The involvement of CK2 activity in malignant cell proliferation makes CK2 a promising druggable target for cancer chemotherapeutics [16]. CK2 activity is fluctuating in cells depending on the cell proliferation stage [11,17] and circadian oscillation [18-20], however CK2 seems constitutively active when isolated. There should be unknown factors that suppress the basal CK2 activity in cells. It is important to unveil at a molecular level how CK2 activity is regulated in cells.

In this study, we investigated the possible link between the NLS and protein phosphorylation. We examined the effect of the NLS-peptide on protein phosphorylation in cell lysates. We revealed that glucose-regulated protein 94 (Grp94), an endoplasmic reticulum (ER)-resident member of the Hsp90 molecular chaperone family, was phosphorylated in an NLS-peptide-dependent manner. The NLS-dependent kinase was identified as CK2, and the NLS-dependency of Grp94 phosphorylation by CK2 was attributable to an additional factor in 
the cell lysates. The physiological importance of the NLS-dependent activation of CK2 is discussed. 


\section{Materials and methods}

Antibodies, proteins, and peptides

Rabbit anti-Grp94 [21] and anti-CK2 $\beta$ [22] antibodies were previously described. The anti-CK2 $\alpha$ antibody was raised against a peptide (CLKPVKKKKIKREIKILENLR) corresponding to the 70-89th amino acid residues of human CK2 $\alpha$ [22]. Grp94 [23] and $\begin{array}{llll}\text { CK2 [24] was purified as described previously. WT-NLS } & \end{array}$ ( $N$-Cys-Pro-Lys-Lys-Arg-Lys-Val-Glu-Asp-Pro-C), MT-NLS

( $N$-Cys-Pro-Lys-Thr-Lys-Arg-Lys-Val-Glu-Asp-Pro-C), and RT-NLS ( $N$-Pro-Asp-Glu-Val-Lys-Arg-Lys-Lys-Lys-Pro-Cys- $C$ ) were chemically synthesized with a model 430A synthesizer (Applied Biosystems) and purified by reverse-phase high performance liquid chromatography. The stock solutions of these peptides were prepared in water.

Preparation of cell lysates

L5178Y and L cells were cultured in DMEM supplemented with 10\% FCS in humidified air containing 5\% $\mathrm{CO}_{2}$. Cells were collected and homogenized in Buffer A (50 mM Hepes, $1 \mathrm{mM}$ dithiothreitol (DTT), $2 \mathrm{mM}$ ethylenediaminetetraacetic acid (EDTA), 10\% glycerol, pH 7.4) containing protease inhibitors ( $1 \mathrm{mM}$ phenylmethylsulfonyl fluoride, $2 \mu \mathrm{g} / \mathrm{mL}$ leupeptine, 1 $\mu \mathrm{g} / \mathrm{mL}$ pepstatin $\mathrm{A}, 10 \mu \mathrm{g} / \mathrm{mL}$ aprotinin). The homogenates were then centrifuged at 100,000 $\times g$ for 30 min at $2{ }^{\circ} \mathrm{C}$ and the supernatants were used as cell lysates.

Large scale purification of the NLS-dependent Grp94 kinase 
L5178Y cells were collected and washed three times with ice-cold PBS, and then ruptured in Buffer A supplemented with the protease inhibitors by a glass-Teflon homogenizer. The homogenate was centrifuged at $150 \mathrm{k} \times g$ for $30 \mathrm{~min}$ at $2{ }^{\circ} \mathrm{C}$ and the supernatant was saved as crude lysate. The lysate was cleared by passing through a $0.22 \mu \mathrm{m}$ low protein binding Durapore-GV filter (Millipore) and applied to a ResourceQ column (GE Healthcare). After extensive washing, bound proteins were eluted with a liner gradient $(0-500 \mathrm{mM}$ of $\mathrm{NaCl})$ in Buffer A. Active fractions were collected and desalted with a Fast Desalting Hitrap column (GE Healthcare). The sample was then charged to a POROS-heparin column (Perseptive POROS 20 HE1) equilibrated in Buffer A. After extensive washing, bound proteins were eluted with a liner gradient $(0-1,000 \mathrm{mM} \mathrm{NaCl})$ in Buffer A. Active fractions were collected, diluted in Buffer $\mathrm{P}$ (20 mM sodium phosphate, $1 \mathrm{mM}$ DTT, $\mathrm{pH}$ 7.2), and applied to a hydroxylapatite column (Pentax SH-0710M). After extensive washing, bound proteins were eluted with a liner gradient of phosphate $(20-1,000 \mathrm{mM})$. Finally, active fractions were collected and applied to a MonoQ column (GE Healthcare) equilibrated in Buffer A and bound proteins were eluted with a liner gradient $(100-1,000 \mathrm{mM} \mathrm{NaCl})$ in buffer $\mathrm{A}$.

Other procedures

CK2 assay using a CK2-specific substrate peptide was performed as described [24]. L cell lysates were mixed with Con A (concanavalin A)-Sepharose affinity resin (GE-Healthcare) and resin-associated Grp94 was removed by a centrifugation to obtain a Con A-flow through (Con A-FT) Grp94-free kinase fraction. Phosphorylation reaction was performed in a solution composed of $10 \mathrm{mM}$ Hepes, $37 \mathrm{mM}$ Tris-Cl, $0.2 \mathrm{mM}$ DTT, $0.4 \mathrm{mM}$ EDTA, 3\% glycerol, $6 \mathrm{mM}$

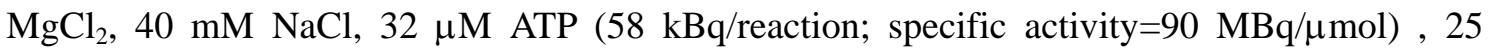
$\mu \mathrm{g} / \mathrm{mL}$ Grp94 (when indicated), $\mathrm{pH} 7.4$, with or without $0.5 \mathrm{mM}$ NLS-peptides. Immunoprecipitation with an anti-Grp94 antibody and V8 mapping of Grp94 were performed 
essentially as described before [23]. Sodium dodecyl sulfate-polyacrylamide gel electrophoresis (SDS-PAGE) and western blotting analysis were performed as described [25]. Silver staining of SDS-polyacrylamide gels was performed using 2D-Silver Stain II kit (Daiichi Pure Chemicals). 


\section{Results}

Induction of phosphorylation of several endogenous protein substrates in cell lysates by an NLS-peptide

L cell lysate was prepared and phosphorylation by endogenous kinases was performed by incubating with $\left[\gamma_{-}{ }^{32} \mathrm{P}\right]$ ATP. In the condition used, we observed only a little phosphorylation of endogenous substrates (Fig. 1a, lane 1). The addition of $0.5 \mathrm{mM}$ of the wild type NLS-peptide (WT-NLS, see Fig. 7a for the sequence) that shows a strong nuclear localization activity induced phosphorylation of four major substrates with molecular masses of 100, 80, 50, and 45 kDa (Fig. 1a, lane 2). MT-NLS is a peptide with a single amino acid replacement in WT-NLS (see Fig. 7a) and it possesses little nuclear localization activity [2,4,5]. RT-NLS is a peptide with a reversed amino acid sequence of WT-NLS (see Fig. 7a) and shows a weak nuclear localization activity [6]. MT-NLS did not stimulate phosphorylation of the four endogenous proteins (Fig. 1a, lane 3) and RT-NLS only weakly stimulated phosphorylation of the same set of endogenous proteins (Fig. 1a, lane 4). The stimulatory effect of these peptides correlated well with the nuclear localization activity of the peptides, suggesting a physiological meaning of the stimulation of phosphorylation of these substrates by the NLS-peptide. The results showed that cell lysates contain an NLS-peptide-dependent kinase.

Identification of the endogenous $100 \mathrm{kDa}$ protein that was phosphorylated by the NLS-dependent protein kinase as Grp94, a glucose-regulated molecular chaperone of the Hsp90 family

Among the four endogenous proteins that underwent NLS-dependent phosphorylation, mobility of pp100 on SDS-PAGE corresponded to an abundant polypeptide in the cell lysates as revealed 
by CBB protein staining of the gel (data not shown). Cytoskeletal proteins and molecular chaperones are the two protein families whose cellular expression is most prominent. We noticed that the gel mobility of pp100 was close to that of major ER-resident glucose regulated molecular chaperone, Grp94. Grp94 (also known as Erp99, gp96, Hsp100, HSP90B1, or endoplasmin) is a member of the major Hsp90 molecular chaperone family and abundant in vertebrate ER. We added purified Grp94 protein in the phosphorylation mixtures of L cell lysates. The mobility of pp100 on SDS-PAGE was exactly the same as that of purified Grp94 (Fig. 1b). In addition, Grp94 exogenously added to the mixture was phosphorylated by an endogenous kinase in a WT-NLS-dependent manner (Fig. 1b, lanes $1 \& 2$ ). The stimulation of phosphorylation of added Grp94 was less significant with MT-NLS or RT-NLS (Fig. 1b, lanes 3 \& 4). The identity of pp100 as Grp94 was then confirmed by immunoprecipitation experiments. L cell lysates were mixed with the peptides in the presence or absence of purified Grp94, and incubated with $\left[\gamma^{32}\right.$ P]ATP. Grp94 in the phosphorylation mixture was then immunoprecipitated with an anti-Grp94 antibody and Protein G-Sepharose. As shown in Fig. 2a, endogenous Grp94 (lanes 1-4) and added purified Grp94 (lanes 5-8) was phosphorylated in an NLS-dependent manner and immunoprecipitated with the anti-Grp94 antibody. These results showed that pp100 is identical with Grp94, and suggested that the cell lysate contains an endogenous kinase that phosphorylates Grp94 in an NLS-peptide-dependent manner.

Grp94 is an ATP-binding protein and possesses autophosphorylation activity [23]. To exclude a possibility that WT-NLS activated Grp94 autophosphorylation rather than phosphorylation by an NLS-dependent kinase, we examined the effect of the NLS-peptides on Grp94 autophosphorylation. When purified Grp94 alone was incubated with $\left[\gamma_{-}{ }^{32} \mathrm{P}\right] \mathrm{ATP}$ in the same condition, we could not detect Grp94 autophosphorylation, and the addition of WT-NLS did not induce autophosphorylation of Grp94 (Fig. 2b, lanes 1-4). Grp94 is a glycoprotein and binds strongly to concanavalin A, so we could remove endogenous Grp94 from the L cell lysate using Con A-Sepharose. The flow-through fraction without Grp94 was used as a source of the 
endogenous kinase and mixed with kinase-free purified Grp94 to reconstitute the phosphorylation reaction. Grp94 was phosphorylated with the Con A-flow through fraction and phosphorylation was stimulated by WT-NLS (Fig. 2b, lane 6), but not by MT-NLS (lane 7) or RT-NLS (lane 8). These results showed that the WT-NLS activated an endogenous kinase and induced phosphorylation of Grp94 in vitro.

Purification of the NLS-dependent Grp94 kinase and its identification as CK2

We then purified the putative NLS-dependent Grp94 kinase from the L5178Y cell lysate by biochemical sequential column chromatography. First, the cell lysate was applied to a Resource Q anion exchange column and each column fraction was tested for its phosphorylation activity to exogenously added Grp94 in the presence and absence of WT-NLS. As shown in Fig. 3a, we detected two Grp94 kinase activity peaks. A kinase activity in fractions 5-9 was rather inhibited by WT-NLS (lanes 1-6). A major peak activity in fractions 15-19 phosphorylated Grp94 in an NLS-peptide-dependent manner (lanes 11-16). The latter fractions were collected and applied to a POROS-heparin column. A major Grp94 kinase activity was detected in fractions 12-15 (Fig. $3 b$ lanes 7-14) and a minor activity was in fraction 6-7 (lanes 1-4). The major peak fractions showed the NLS-dependency. The major peak fractions were collected and the Grp94 kinase was further purified on a hydroxylapatite column (Fig. 3c) and finally on a MonoQ anion exchange column (Fig. 3d). In the last step, a sharp single peak of Grp94 phosphorylating activity was detected in fraction 9 (Fig. 3d, lane 7). The protein profile of the purified Grp94 kinase in MonoQ column fractions was shown by silver staining in Fig. 4a. The purified kinase in peak fraction 9 (lane 4) was composed of two major polypeptides with molecular masses of $42 \mathrm{kDa}$ and $27 \mathrm{kDa}$. The molecular masses of these polypeptides corresponded to that of $\mathrm{CK} 2 \alpha$ and CK2 $\beta$. Indeed, western blotting analysis showed that the $42 \mathrm{kDa}$ and $27 \mathrm{kDa}$ polypeptides were recognized by anti-CK2 $\alpha$ and anti-CK2 $\beta$ 
antibodies, respectively (Fig. 4b). The peptide-directed anti-CK2 $\alpha$ antibody might also recognize $\mathrm{CK} 2 \alpha^{\prime}$, hence, a minor band with a molecular mass of $40 \mathrm{kDa}$ detected by silver staining and on the western blot could be CK2 $\alpha^{\prime}$ or a degradation product of CK2 2 . We then re-evaluated CK2 activity through all the column chromatography steps by a peptide-directed in vitro CK2 specific assay (Fig. 5). The CK2 activity peak fraction numbers (fraction 17 in Resource Q, fraction 14 in POROS-heparin, fraction 9 in hydroxylapatite, fraction 9 in Mono Q) were exactly identical with fraction numbers of the Grp94 kinase peaks (Fig. 3).

CK2 is known to use GTP as a phosphate donor as comparable as ATP, which is one of the CK2-specific characteristics. We examined the effect of an excess amount of GTP on ATP-driven phosphorylation of Grp94 in the cell lysates. GTP effectively suppressed NLS-dependent phosphorylation of both endogenous (lanes 1-4) and exogenous (lanes 5-8) Grp94 (Fig. 6a). In Fig. 6b, the phosphorylation sites of Grp94 were analyzed by V8 peptide mapping. Purified Grp94 was phosphorylated by purified CK2 (lanes 1-4) or by the NLS-dependent kinase in the cell lysates (Con A-flow through fraction) (lanes 5-8). Both kinases gave an indistinguishable V8 fragment pattern (Fig. 6b, compare lanes $3 \& 4$ to lanes 7 \& 8). All of the silver staining profile, the western blotting analysis, the CK2 activity column profiles, GTP effect, and the V8 mapping experiments, altogether indicated that the purified Grp94 kinase is identical with CK2.

NLS-dependent phosphorylation of Grp94 by CK2 requires an additional factor

While CK2 in cell lysates phosphorylated Grp94 in an NLS-dependent manner (Fig. 6b, compare lanes $5 \& 6$ with lanes $7 \&$ 8), we noticed that purified CK2 phosphorylated Grp94 even without the NLS-peptide (Fig. 6b, lanes 1-4). Indeed, when we incubated purified Grp94 with purified CK2 holoenzyme, CK2 phosphorylated Grp94 without the WT-NLS peptide (Fig. 6c, lane 5), and WT-NLS, MT-NLS, or RT-NLS did not stimulate phosphorylation of Grp94 by 
CK2 (Fig. 6c, lanes 5-8, compare with Fig. 2b). In the final MonoQ column chromatography step, CK2 in the peak fractions 8-10 phosphorylated Grp94 in a WT-NLS-independent manner (Fig. 6d). Moreover, phosphorylation of several CK2 substrates, Hsp90, casein, and the CK2-substrate peptide, as well as autophosphorylation of CK2 in its $\beta$ subunit, was not affected by the NLS-peptide (data not shown). Therefore, WT-NLS should not be a direct activator of CK2. We concluded that purified CK2 holoenzyme phosphorylated Grp94 in vitro in an NLS-independent manner, whereas phosphorylation of Grp94 by CK2 was suppressed in cells and WT-NLS stimulated CK2-dependent Grp94 phosphorylation by releasing the suppression. There should be an additional factor in cells that is required for the NLS-dependency of Grp94 phosphorylation by CK2. 


\section{Discussion}

In this study, we found that a peptide with a nuclear localization activity stimulated phosphorylation of an ER molecular chaperone Grp94 by an endogenous kinase in cell lysates. Evidence indicated that the kinase responsible for NLS-dependent phosphorylation of Grp94 is identical with CK2, a ubiquitous and evolutionary-conserved pleiotropic protein kinase composed of two $\alpha$ and two $\beta$ subunits. Surprisingly, pure CK2 holoenzyme phosphorylated Grp94 efficiently, however, in an NLS-independent manner. This suggests that an additional suppressive factor in the cell lysate is required for the NLS-dependency of Grp94 phosphorylation by CK2.

Grp94 belongs to the Hsp90 molecular chaperone family and shares a significant sequence similarity with Hsp90. However, Grp94 is different from Hsp90 in many respects [26]. The intracellular localization of Hsp90 is mainly cytoplasmic, while Grp94 localizes in the ER [26]. The amino acid sequence of the C-terminal end of Hsp90 stops with 'EEVD', which is essential for its interaction with TPR (tetratricopeptide repeat)-containing co-chaperones [27]. By contrast, the amino acid sequence of the C-terminal end of Grp94 is 'KDEL' that is required for the retention of proteins in the ER [28]. Hsp90 functions in cooperation with many co-chaperones, including Cdc37, FKBPs, cyclophilins, and p23 [29], whereas no co-chaperone for Grp94 was so far identified. The list of the client proteins of Hsp90 includes many cytosolic and nuclear signaling molecules such as protein kinases and transcription factors [30], while the binding partners for Grp94 are proteins in the ER-resident protein quality controlling complexes [26]. Despite these differences, both proteins share an ability to bind to CK2 and to be phosphorylated by CK2. Hsp90 binds to the $\alpha$ catalytic subunit of CK2 [22] and protects CK2 from aggregating [24]. In addition, CK2 phosphorylates Hsp90 on several sites and modulates the molecular chaperone activity of Hsp90 [15,31,32]. 
Two major tryptic phosphopeptides from CK2-catalyzed canine Grp94 contained Ser306 and Thr786 [33]. In addition, Grp94 was shown to be a substrate of CK2 in cells [34]. Proteomic mass spectrometry approaches identified several phosphorylation sites of Grp94 in cells, including the same residues, Ser306 (pSDDEAAVE) [35] and Thr786 (pTDEEEETA) [36]. Both the phosphorylation sites agree nicely with the phosphorylation motif for CK2, so Grp94 should be phosphorylated by CK2 both in vitro and in vivo mainly on Ser306 and Thr786. The functional regulation of Grp94 by phosphorylation, however, has not been precisely revealed. Moreover, it remains unclear whether phosphorylation on both sites by CK2 is stimulated by the NLS-peptide.

Intimate functional relationship between Grp94 and CK2 has previously been suggested. Physical association and co-purification of CK2 with Grp94 were reported [23,37], and CK2 $\alpha$ binds to the C-terminal domain of Grp94 [38]. The binding of the C-terminal domain of Grp94 protects CK2 $\alpha$ from aggregation [39]. This effect is reminiscent of the protective role for Hsp90 toward CK2 [22,24].

The NLS of SV40 Large T antigen is a short sequence of a single series of basic residues. CK2 is known to be activated by stretches of basic amino acids such as polylysine and polyarginine [11-14]. The NLS-peptide might thus activate CK2 just as polylysine or polyarginine do. Indeed, phosphorylation of sarcoreticulum proteins including Grp94 by CK2 was enhanced by the addition of polylysine [33]. However, our results suggested that this might not be the case, because the NLS-peptide activated CK2 only in the cell lysates, but purified CK2 could not be activated by the NLS-peptide. An additional suppressive factor in the cell lysate is required for the observed activation of CK2 by the NLS-peptide. Moreover, RT-NLS possesses the same amino acid composition as WT-NLS, but could not stimulate Grp94 phosphorylation by CK2.

The physiological importance of observed NLS-dependent phosphorylation of Grp94 by CK2 remains unelucidated in this study. Phosphorylation in the vicinity of the NLS plays a role in 
regulating nuclear protein import through modulation of NLS function in either a positive or negative way $[10,40]$. CK2-dependent phosphorylation in the region near the NLS in SV40 Large $\mathrm{T}$ antigen (position -13 , see Fig. $7 \mathrm{~b}$ ) was reported to enhance the binding of importin family NLS receptors and increase the nuclear translocation of the protein $[41,42]$. In many other nuclear proteins, including polyoma $\mathrm{T}$ antigen, nucleoplasmin, lamin $\mathrm{A} / \mathrm{C}, \mathrm{c}-\mathrm{Myc}, \mathrm{Ad} 7$ E1a, p53, and NF- $\mathrm{B}$ p50, there is a CK2 phosphorylation site in the vicinity of the NLS [43]. Our results in this study suggest that the NLS in a nuclear protein interacts transiently with CK2 with a help of the putative suppressive factor $\mathrm{X}$ in cells (see Fig. 7c) and thereby the basic amino acid stretch in the NLS sequence activates associated CK2. CK2 might then efficiently phosphorylate the nearby CK2-site in the same protein complex. After the dissociation of CK2 by phosphorylation, importin family proteins may recognize and bind efficiently to the NLS adjacent to the CK2-catalyzed phosphorylation site. This may be one of the molecular mechanism by which CK2 (and possibly other protein kinases) plays a role in positively or negatively controlling the efficiency of nuclear translocation. Phosphorylation of another set of protein substrates was previously shown to be stimulated in Xenopus oocyte and in Ehrlich ascites tumor cells by a different NLS-peptide [44], and the kinase responsible for this phosphorylation seems to be different from CK2 [44]. In conclusion, our results suggest a possible role for CK2 in controlling nuclear protein translocation by unique NLS-dependent phosphorylation of a CK2 site in the vicinity of the NLS sequence in nuclear proteins. Identification of the additional factor that confers NLS-dependency to CK2 is matters for future investigation. 
Acknowledgements We thank Professor Eisuke Nishida for his support and encouragement. We would like to give our thanks to K. Kimura, H. Izumi, and T. Sakabe for their excellent technical assistance. This work was supported by Grants-in-Aid for Scientific Research from the Ministry of Education, Culture, Sports, Science and Technology of Japan. 


\section{References}

1. Pollard VW, Michael WM, Nakielny S, Siomi MC, Wang F, Dreyfuss G (1996) A novel receptor-mediated nuclear protein import pathway. Cell 86:985-994

2. Kalderon D, Richardson WD, Markham AF, Smith AE (1984) Sequence requirements for nuclear location of simian virus 40 large-T antigen. Nature 311:33-38

3. Dingwall C, Sharnick SV, Laskey RA (1982) A polypeptide domain that specifies migration of nucleoplasmin into the nucleus. Cell 30:449-458

4. Kalderon D, Roberts BL, Richardson WD, Smith AE (1984) A short amino acid sequence able to specify nuclear location. Cell 39:499-509

5. Goldfarb DS, Gariepy J, Schoolnik G, Kornberg RD (1986) Synthetic peptides as nuclear localization signals. Nature 322:641-644

6. Adam SA, Gerace L (1991) Cytosolic proteins that specifically bind nuclear location signals are receptors for nuclear import. Cell 66:837-847

7. Lange A, Mills RE, Lange CJ, Stewart M, Devine SE, Corbett AH (2007) Classical nuclear localization signals: definition, function, and interaction with importin $\alpha$. J Biol Chem 282:5101-5105

8. Mosammaparast N, Pemberton LF (2004) Karyopherins: from nuclear-transport mediators to nuclear-function regulators. Trends Cell Biol 14:547-556

9. Görlich D, Mattaj IW (1996) Nucleocytoplasmic transport. Science 271:1513-1518

10. Jans DA, Hübner S (1996) Regulation of protein transport to the nucleus: central role of phosphorylation. Physiol Rev 76:651-685

11. Pinna LA, Meggio F (1997) Protein kinase CK2 ("casein kinase-2") and its implication in cell division and proliferation. Prog Cell Cycle Res 3:77-97

12. Litchfield DW (2003) Protein kinase CK2: structure, regulation and role in cellular decisions of life and death. Biochem J 369:1-15 
13. Guerra B, Boldyreff B, Sarno S, Cesaro L, Issinger OG, Pinna LA (1999) CK2: a protein kinase in need of control. Pharmacol Ther 82:303-313

14. Meggio F, Pinna LA (2003) One-thousand-and-one substrates of protein kinase CK2? FASEB J 17:349-368

15. Miyata Y (2009) CK2: the kinase controlling the Hsp90 chaperone machinery. Cell Mol Life Sci 66:1840-1849

16. Ahmad KA, Wang G, Slaton J, Unger G, Ahmed K (2005) Targeting CK2 for cancer therapy. Anticancer Drugs 16:1037-1043

17. Filhol-Cochet O, Loue-Mackenbach P, Cochet C, Chambaz EM (1994) Casein kinase 2 and the cell response to growth factors. Cell Mol Biol Res 40:529-537

18. Allada R, Meissner RA (2005) Casein kinase 2, circadian clocks, and the flight from mutagenic light. Mol Cell Biochem 274:141-149

19. Blau J (2003) A new role for an old kinase: CK2 and the circadian clock. Nat Neurosci 6:208-210

20. Tsuchiya Y, Akashi M, Matsuda M, Goto K, Miyata Y, Node K, Nishida E (2009) Involvement of the protein kinase CK2 in the regulation of mammalian circadian rhythms. Sci Signal 2:ra26

21. Takemoto H, Yoshimori T, Yamamoto A, Miyata Y, Yahara I, Inoue K, Tashiro Y (1992) Heavy chain binding protein (BiP/GRP78) and endoplasmin are exported from the ER in rat exocrine pancreatic cells, similar to protein disulfide-isomerase. Archiv Biochem Biophys 296:129-136

22. Miyata Y, Yahara I (1995) Interaction between casein kinase II and the 90-kDa stress protein, HSP90. Biochemistry 34:8123-8129

23. Csermely P, Miyata Y, Schnaider T, Yahara I (1995) Autophosphorylation of grp94 (Endoplasmin). J Biol Chem 270:6381-6388 
24. Miyata Y, Yahara I (1992) The 90-kDa heat shock protein, HSP90, binds and protects casein kinase II from self-aggregation and enhances its kinase activity. J Biol Chem 267:7042-7047

25. Miyata Y, Nishida E (2004) CK2 controls multiple protein kinases by phosphorylating a kinase-targeting molecular chaperone Cdc37. Mol Cell Biol 24:4065-4074

26. Eletto D, Dersh D, Argon Y (2010) GRP94 in ER quality control and stress responses. Semin Cell Dev Biol 21:479-485

27. Pratt WB (1998) The hsp90-based chaperone system: involvement in signal transduction from a variety of hormone and growth factor receptors. Proc Soc Exp Biol Med $217: 420-434$

28. Munro S, Pelham HR (1987) A C-terminal signal prevents secretion of luminal ER proteins. Cell 48:899-907

29. Picard D (2002) Heat-shock protein 90, a chaperone for folding and regulation. Cell Mol Life Sci 59:1640-1648

30. Richter K, Buchner J (2001) Hsp90: Chaperoning signal transduction. J Cell Physiol $188: 281-290$

31. Szyszka R, Kramer G, Hardesty B (1989) The phosphorylation state of the reticulocyte 90-kDa heat shock protein affects its ability to increase phosphorylation of peptide initiation factor $2 \alpha$ subunit by the heme-sensitive kinase. Biochemistry 28:1435-1438

32. Mollapour M, Tsutsumi S, Truman AW, Xu W, Vaughan CK, Beebe K, Konstantinova A, Vourganti S, Panaretou B, Piper PW, Trepel JB, Prodromou C, Pearl LH, Neckers L (2010) Threonine 22 phosphorylation attenuates hsp90 interaction with cochaperones and affects its chaperone activity. Mol Cell 41:672-681

33. Cala SE, Jones LR (1994) GRP94 resides within cardiac sarcoplasmic reticulum vesicles and is phosphorylated by casein kinase II. J Biol Chem 269:5926-5931 
34. Cala SE (2000) GRP94 hyperglycosylation and phosphorylation in Sf21 cells. Biochim Biophys Acta 1496:296-310

35. Bennetzen MV, Larsen DH, Bunkenborg J, Bartek J, Lukas J, Andersen JS (2010) Site-specific phosphorylation dynamics of the nuclear proteome during the DNA damage response. Mol Cell Proteomics 9:1314-1323

36. Old WM, Shabb JB, Houel S, Wang H, Couts KL, Yen CY, Litman ES, Croy CH, Meyer-Arendt K, Miranda JG, Brown RA, Witze ES, Schweppe RE, Resing KA, Ahn NG (2009) Functional proteomics identifies targets of phosphorylation by B-Raf signaling in melanoma. Mol Cell 34:115-131

37. Riera M, Roher N, Miro F, Gil C, Trujillo R, Aguilera J, Plana M, Itarte E (1999) Association of protein kinase CK2 with eukaryotic translation initiation factor eIF-2 and with grp94/endoplasmin. Mol Cell Biochem 191:97-104

38. Roher N, Miro F, Boldyreff B, Llorens F, Plana M, Issinger OG, Itarte E (2001) The C-terminal domain of human grp94 protects the catalytic subunit of protein kinase CK2 (CK2 $\alpha$ ) against thermal aggregation. Role of disulfide bonds. Eur $\mathrm{J}$ Biochem $268: 429-436$

39. Roher N, Sarno S, Miro F, Ruzzene M, Llorens F, Meggio F, Itarte E, Pinna LA, Plana M (2001) The carboxy-terminal domain of Grp94 binds to protein kinase CK2 $\alpha$ but not to CK2 holoenzyme. FEBS Lett 505:42-46

40. Fontes MR, Teh T, Toth G, John A, Pavo I, Jans DA, Kobe B (2003) Role of flanking sequences and phosphorylation in the recognition of the simian-virus-40 large T-antigen nuclear localization sequences by importin- $\alpha$. Biochem J 375:339-349

41. Hübner S, Xiao CY, Jans DA (1997) The protein kinase CK2 site (Ser111/112) enhances recognition of the simian virus 40 large T-antigen nuclear localization sequence by importin. J Biol Chem 272:17191-17195 
42. Xiao CY, Jans P, Jans DA (1998) Negative charge at the protein kinase CK2 site enhances recognition of the SV40 large T-antigen NLS by importin: effect of conformation. FEBS Lett 440:297-301

43. Rihs HP, Jans DA, Fan H, Peters R (1991) The rate of nuclear cytoplasmic protein transport is determined by the casein kinase II site flanking the nuclear localization sequence of the SV40 T-antigen. EMBO J 10:633-639

44. Kurihara T, Hori M, Takeda H, Inoue M, Yoneda Y (1996) Partial purification and characterization of a protein kinase that is activated by nuclear localization signal peptides. FEBS Lett 380:241-245 


\section{Figure legends}

Fig. 1 (a) NLS-peptide-dependent activation of a protein kinase that phosphorylates endogenous substrates. Phosphorylation of proteins by endogenous kinases in L cell lysates was performed in the presence of WT-NLS (lane 2), MT-NLS (lane 3), or RT-NLS (lane 4). As a control, no peptide was added (lane 1). (b) NLS-dependent phosphorylation of purified Grp94 by an endogenous kinase. Purified Grp94 was mixed with L cell lysates and phosphorylation was performed in the presence of WT-NLS (lane 2), MT-NLS (lane 3), or RT-NLS (lane 4). Phosphorylation reaction was performed without a peptide (lane 1) or exogenous Grp94 (lane 5). The positions of four major phosphoproteins analyzed by SDS-PAGE and autoradiography (pp100, pp80, pp50, and pp45 according to their apparent molecular masses) are shown (a \& b).

Fig. 2 Identification of the pp100 substrate for the NLS-dependent kinase as Grp94. (a) Immunoprecipitation of Grp94 after phosphorylation. L cell lysates were incubated with $\left[\gamma-{ }^{32} \mathrm{P}\right] \mathrm{ATP}$ in the absence (lanes 1-4) or presence (lanes 5-8) of purified Grp94. Phosphorylation was performed in the presence of WT-NLS (lanes $2 \& 6$ ), MT-NLS (lanes $3 \&$ 7), or RT-NLS (lanes 4 \& 8). As controls, no peptide was included (lanes 1 \& 5). After phosphorylation, Grp94 was immunoprecipitated and analyzed by SDS-PAGE and autoradiography. (b) Reconstitution of NLS-dependent phosphorylation of Grp94. Purified Grp94 was incubated alone (lanes 1-4) or with a Con A-flow through fraction of L cell lysate as a source of the kinase (lanes 5-8) in the presence of $\left[\gamma^{32}\right.$ P $]$ ATP and analyzed by SDS-PAGE and autoradiography. WT-NLS (lanes $2 \& 6$ ), MT-NLS (lanes $3 \&$ 7), or RT-NLS (lanes 4 \&8) was included in the mixture. As controls, no peptide was added (lanes $1 \& 5$ ). 
Fig. 3 Purification of the NLS-dependent Grp94 kinase. The protein kinase that phosphorylates Grp94 in the presence of WT-NLS was purified by sequential column chromatography on (a) Resource Q; (b) POROS-heparin; (c) hydroxylapatite; (d) MonoQ. The Grp94 phosphorylating activity in each fraction was evaluated by mixing fractions with purified Grp94 and $\left[\gamma^{32} \mathrm{P}\right]$ ATP, and analyzed by SDS-PAGE and autoradiography. In (a) and (b), the Grp94 phosphorylation activity in each fraction was monitored with (+) or without (-) the WT-NLS peptide.

Fig. 4 Identification of the NLS-dependent Grp94 kinase as CK2. (a) Silver staining of the purified Grp94 kinase in MonoQ fractions separated by SDS-PAGE. The positions of two major polypeptides (42 kDa and $27 \mathrm{kDa}$ ) in fraction 9 (lane 4) are indicated. (b) Western blotting analysis of the polypeptides in the MonoQ fraction 9 with an anti-CK2 $\alpha$ (lane 1) or an anti-CK2 $\beta$ (lane 2) antibody.

Fig. 5 CK2 activity assay in the sequential column chromatography steps. CK2 activity in each fraction in the column chromatography steps for purification of the Grp94 kinase was determined with a CK2-specific substrate peptide. The results are indicated as \% activity of the peak fraction. (a) ResourceQ; (b) POROS-heparin; (c) hydroxylapatite; (d) MonoQ. ' 'O': Original mixture before separation on the column; 'F': Flow-through fraction; 'W': Wash fraction. The fraction number in each column chromatography step with the peak activity is underlined.

Fig. 6 Phosphorylation of Grp94 by CK2. (a) Effect of an excess amount of GTP on NLS-dependent Grp94 phosphorylation. $\left[\gamma_{-}{ }^{32} \mathrm{P}\right] \mathrm{ATP}$ and $\mathrm{L}$ cell lysates containing the NLS-dependent Grp94 kinase were incubated with purified Grp94 (lanes 5-8) or alone (lanes 1-4) in the absence (lanes $1,2,5 \& 6$ ) or presence (lanes $3,4,7 \& 8$ ) of WT-NLS. Cold GTP 
(3 $\mathrm{mM}$ ) was included in the phosphorylation mixtures (lanes 2, 4, 6, \& 8). (b) V8 peptide mapping of Grp94 phosphorylated by purified CK2 (lanes 1-4) or by the NLS-dependent Grp94 kinase (Con A flow-through fraction, lanes 5-8). The amount of V8 protease used was $1 \mu \mathrm{g}$ (lanes $1,3,5 \& 7$ ) or $10 \mu \mathrm{g}$ (lanes $2,4,6 \& 8$ ). WT-NLS was included in the phosphorylation mixtures (lanes 3, 4, 7 \& 8). (c) Phosphorylation of Grp94 by purified CK2 holoenzyme. Purified Grp94 was incubated alone (lanes 1-4) or with purified CK2 (lanes 5-8). WT-NLS (lanes 2 \& 6), MT-NLS (lanes $3 \&$ 7), or RT-NLS (lanes 4 \& 8) was included in the phosphorylation mixtures. (d) NLS-independent phosphorylation of Grp94 by MonoQ column chromatography fractions. Fractions 7-11 of the final MonoQ column chromatography step were assayed for the Grp94 phosphorylation activity with (lanes $2,4,6,8 \& 10$ ) or without (lanes 1, 3, 5, 7 \& 9) WT-NLS. Phosphorylation of Grp94 (a, c, d) or its V8 fragments (b) was analyzed by SDS-PAGE and autoradiography.

Fig. 7 (a) Amino acid sequences of WT-NLS, MT-NLS, and RT-NLS, and their nuclear localization potency. (b) The amino acid sequence of SV40 Large T antigen in the region containing the CK2 phosphorylation site and the NLS. The CK2 phosphorylation site is red-underlined in a blue box, and the NLS sequence is marked by a pink box. (c) The schematic illustration showing a possible role for CK2 in phosphorylation of a common CK2 site near the NLS sequence on a nuclear protein. Binding affinity of importin to the NLS of the protein is enhanced by phosphorylation that is catalyzed by NLS-stimulated CK2. 
(a)

(b)

L Cell Lysate

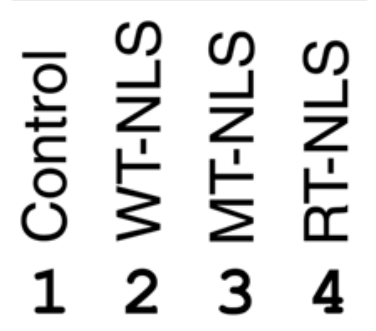

-pp100

-pp80

-pp50

ᄂpp45

Autoradiography

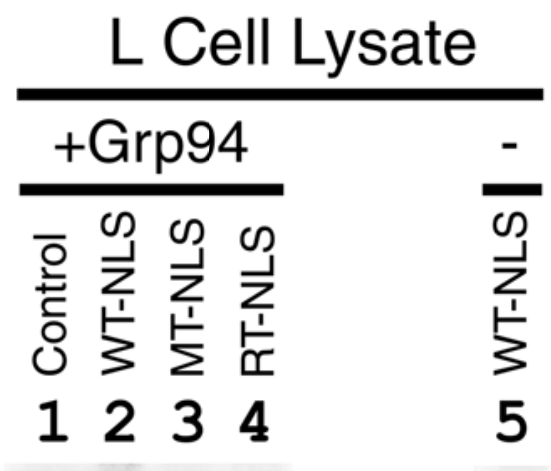

Autoradiography

Fig. 1 
(a) Immunoprecipitation (Anti-Grp94)

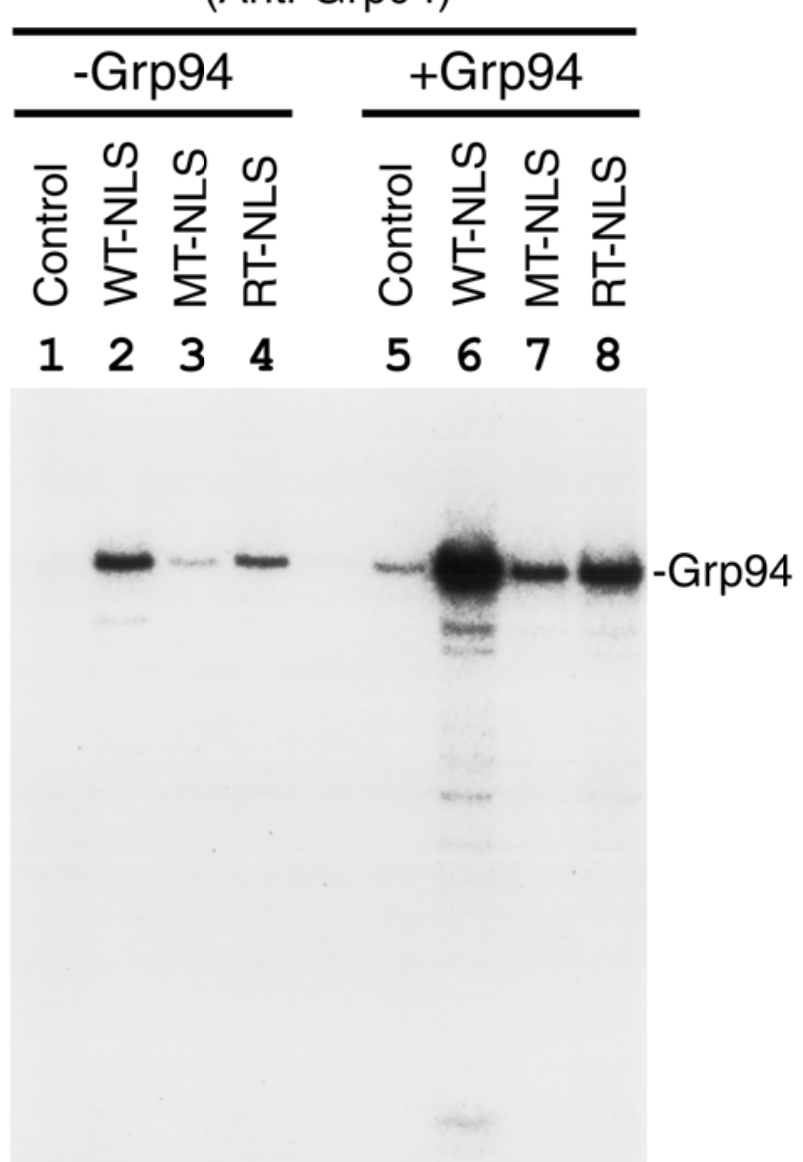

Autoradiography (b)

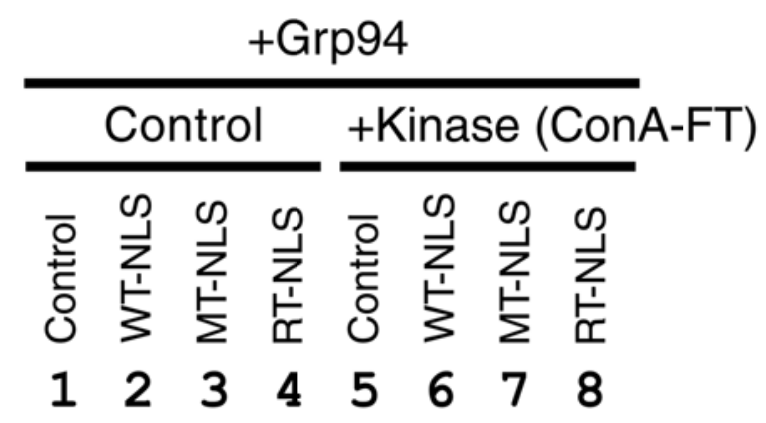

\section{Autoradiography}

Fig. 2 
(a)

\begin{tabular}{|c|c|c|c|c|c|c|c|c|c|}
\hline ction: & 5 & 7 & 9 & 11 & 13 & 15 & 17 & 19 & 21 \\
\hline
\end{tabular}

Grp94- - - - -

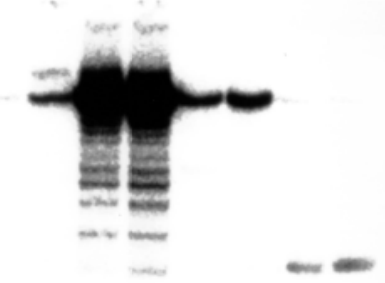

(b)

Autoradiography

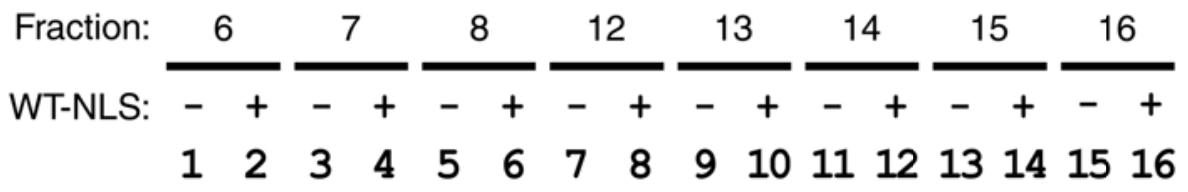

Grp94-

Autoradiography

(c)

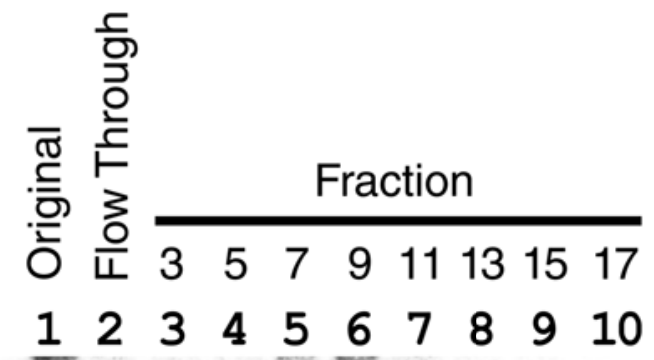

Grp94- (d)

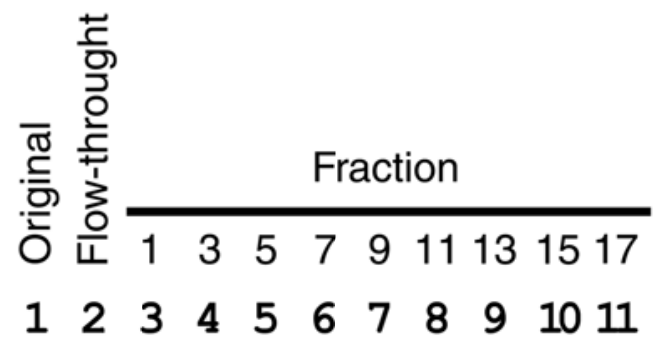

Grp94-

Autoradiography

Fig. 3 


\section{(a) Silver Stain}

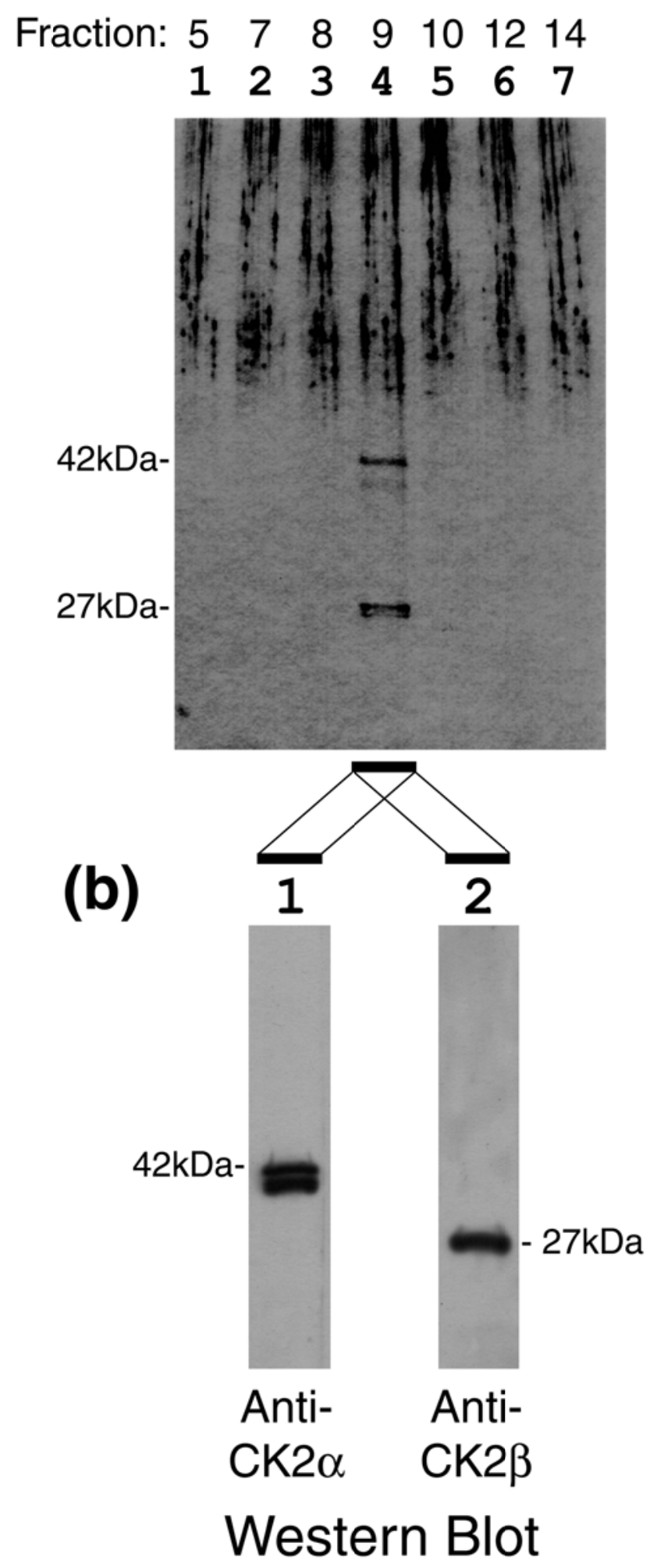

Fig. 4 

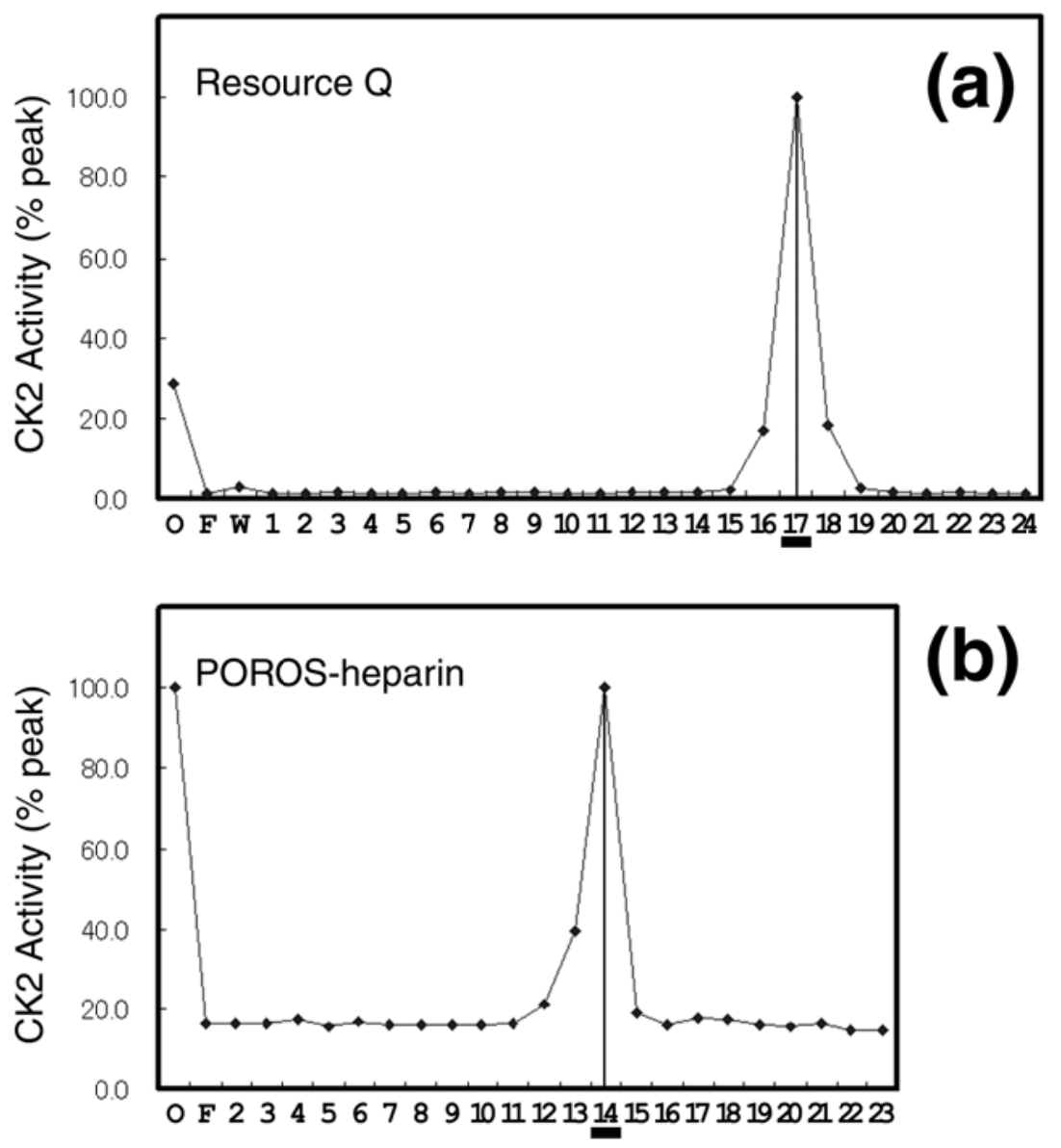

(b)

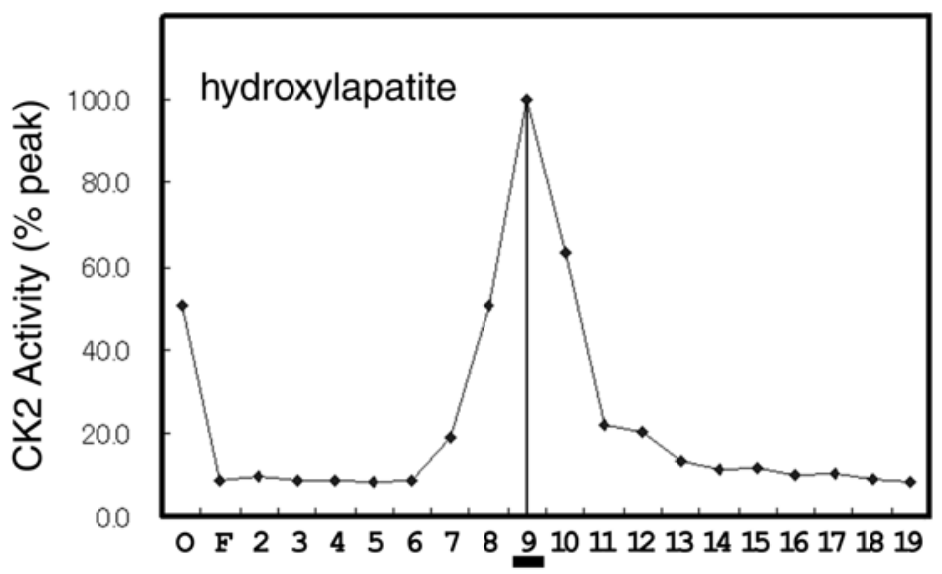

(c)

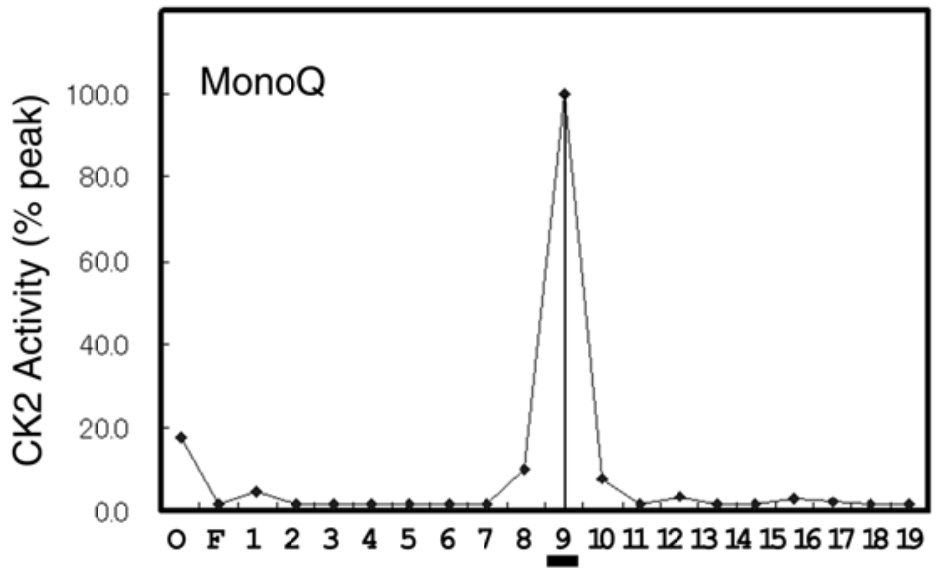

(d)

Fig. 5 
(a)

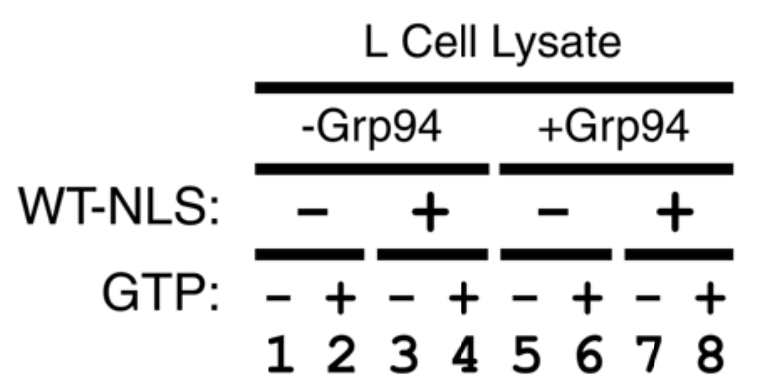

Grp94- (b)

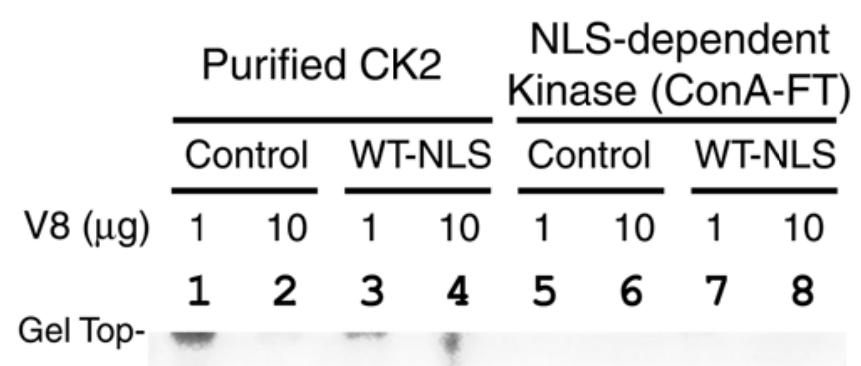


(a)

\begin{tabular}{rcl} 
Peptide Name & $\begin{array}{c}\text { Amino Acid } \\
\text { Sequence }\end{array}$ & $\begin{array}{c}\text { Nuclear Translocation } \\
\text { Activity }\end{array}$ \\
\hline WT-NLS & CPKKKRKVEDP & Strong \\
MT-NLS & CPKTKRKVEDP & Very weak \\
RT-NLS & PDEVKRKKKPC & Weak
\end{tabular}

(b)

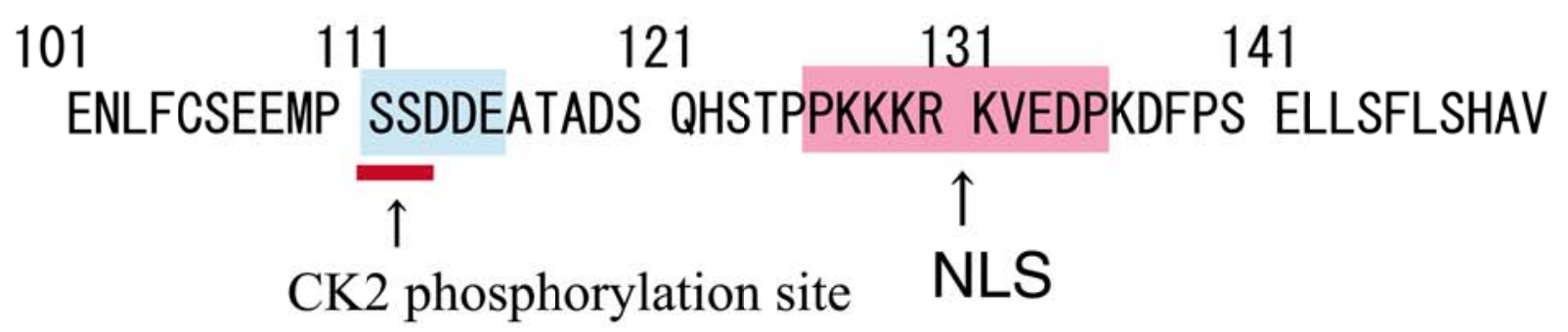

(c)
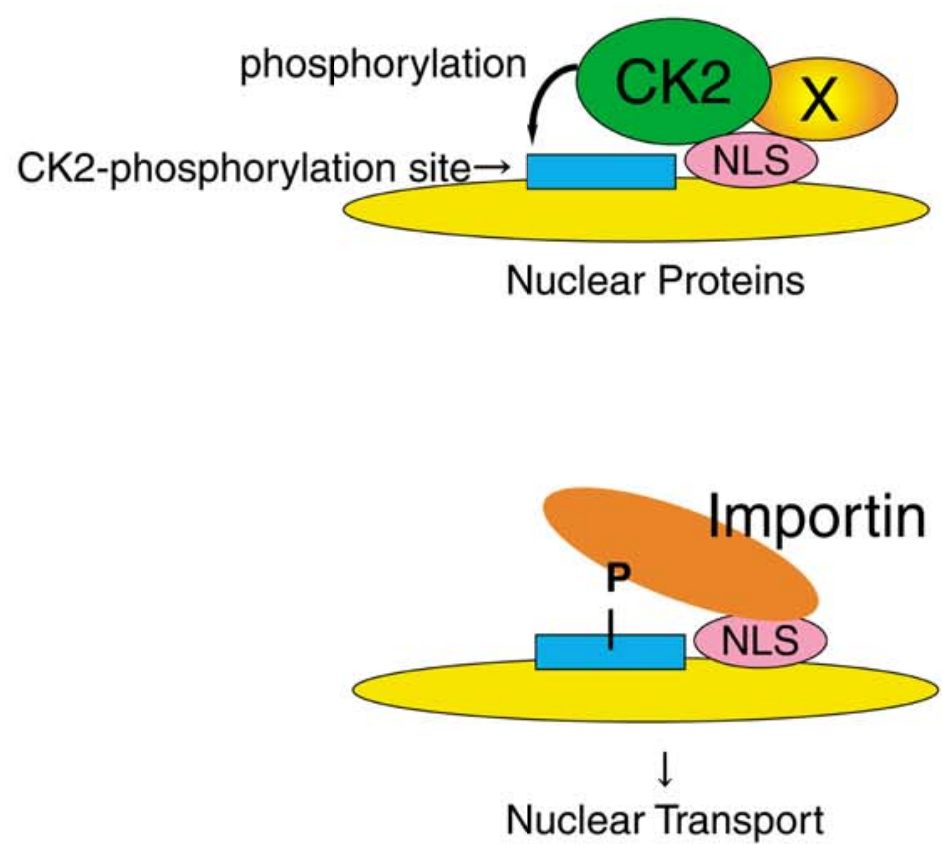

Fig. 7 